\title{
Perfil metabólico de riesgo cardiovascular y resistencia a la insulina según índice de masa corporal, circunferencia de cintura y cintura hipertrigliceridémica en pacientes adultos
}

\author{
Milagros Espinoza Z1,5a, Nelina Ruiz F2,6a, Emilia Barrios ${ }^{3,5 a}$, \\ Aldo Reigosa ${ }^{1,6}$, U lises Leal $\mathbf{H}^{4}$, Julio César G onzález ${ }^{1,6 a}$. \\ Cardiovascular risk profile \\ and insulin resistance according body \\ mass index, waist circumference \\ and hypertriglyceridemic waist \\ in adult subjects
}

\begin{abstract}
Background: Insulin plays a determinant role in the individual metabolic profile. Obesity, the most common cause of insulin resistance (IR), can be diagnosed by body mass index (BMI). Waist circumference (WC), specifically, measures accumulation of abdominal fat. The cardiovascular risk of an individual is strongly related to a combination of abdominal obesity with hypertriglyceridemia or hypertriglyceridemic waist (HTW). Aim: To assess the metabolic profile of cardovascular risk and RI according to BMI, WC and HTW in adult patients. Material and methods: Weight, height, BMI, blood pressure (BP), WC, HTW, blood glucose, total cholesterol, HDL cholesterol, LDL cholesterol, triglycerides and insulin were assessed and IR calculated by the homeostasis model assessment (HOMA) in 63 females aged $46 \pm 11$ years and 12 males aged $47 \pm 10$ years. Results: Differences in weight, WC, BP, lipids, glucose, insulin and HOMA, were observed as BMI increased. When patients were grouped according to WC, there were significant differences only in systolic pressure, triglycerides, insulin and HOMA. Individuals with HTW had a more adverse metabolic profile than those without HTW or with only high WC. A multiple regression equation determined that body mass index, total cholesterol and diastolic blood pressure were independent predictors of insulin resistance. Conclusions: Patients with HTW displayed an unfavorable cardiac risk profile (Rev Méd Chile 2009; 137: 1179-86).
\end{abstract}

(Key w ords: Hypertriglyceridemia; Insulin resistance; Obesity)

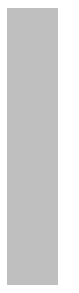

\begin{abstract}
Recibido el 22 de septiembre, 2008. Aceptado el 27 de julio, 2009
${ }^{1}$ Centro de Investigaciones Médicas y Biotecnológicas de la Universidad de Carabobo (CIMBUC), Facultad de Ciencias de la Salud, Universidad de Carabobo, Venezuela. ${ }^{2}$ Centro de Investigaciones en Nutrición (CEINUT), Facultad de Ciencias de la Salud, Universidad de Carabobo, Venezuela. ${ }^{3}$ Centro de Biología Molecular de Parásitos (BioMolP), Facultad de Ciencias de la Salud, Universidad de Carabobo, Venezuela. ${ }^{4}$ Unidad de Atención Médico Integral de la Universidad de Carabobo (UAMI), Venezuela. Departamentos de Investigación y Desarrollo Profesional ${ }^{5}$ y de Morfofisiopatología ${ }^{6}$, Escuela de Bioanálisis, Universidad de Carabobo, Venezuela. ${ }^{a}$ Licenciado en Bioanálisis
\end{abstract}

Correspondencia a: Milagros Espinoza Zavala. Conjunto Residencial Tazajal. Torre Sur. Apto 8F. Urb. Tazajal, Naguanagua. Edo. Carabobo, Venezuela. Código Postal: 2001. Fono: +58 [241] 8919068. Fax: +58 [241] 8919068, E mail: mespinoza@uc.edu.ve; eszami@hotmail.com 
$\mathrm{L}$ a insulina ejerce un papel primordial como regulador de la homeostasis de la glucosa y los lípidos, por lo tanto interviene en el perfil metabólico del individuo ${ }^{1}$. Una disminución de la sensibilidad tisular a la insulina provoca un incremento compensatorio de la secreción de la hormona que se conoce como resistencia a la insulina (RI), un trastorno cuya principal causa en el humano, es la obesidad $^{1}$.

La obesidad puede ser diagnosticada a través de un método de fácil medición y universalmente aceptado, llamado índice de masa corporal $(\mathrm{IMC})^{2}$. Específicamente, la acumulación anormal de grasa en la región abdominal, se denomina centrípeta y la variable antropométrica más utilizada para su estimación es la medida de la circunferencia de cintura $(\mathrm{CC})^{3}$.

Es bien aceptado que la obesidad constituye un factor de riesgo para la salud debido a su asociación con numerosas complicaciones metabólicas ${ }^{4}$. Estudios epidemiológicos han descrito que existe un aumento de la incidencia de ciertas enfermedades crónicas no transmisibles con el aumento del IMC, pero desde el punto de vista clínico, esta definición tiene algunas limitaciones, dado que algunos pacientes pueden presentar un perfil metabólico de riesgo normal a pesar de mostrar un exceso de grasa corporal, mientras que en otros con un exceso moderado de peso, se observan complicaciones metabólicas ${ }^{5,6}$. Ante esto, diversas investigaciones han enfatizado la noción introducida por Vague en $1947^{7}$, que indicaba que las personas que presentaban una obesidad de tipo abdominal desarrollaban diversas patologías cardiometabólicas, más frecuentemente que aquellas con obesidad periférica ${ }^{8}$.

Por otra parte, se ha evidenciado en hombres de mediana edad inicialmente asintomáticos, la presencia de un nuevo fenotipo en el cual converge una tríada de marcadores metabólicos de riesgo aterogénico: hiperinsulinemia en ayunas, incremento de la apolipoproteína B y las LDL pequeñas y densas9. Dado que estas determinaciones son costosas y poco accesibles, investigaciones han demostrado que esta tríada tiene un altísimo porcentaje de concordancia con la díada: CC elevada más hipertrigliceridemia, conjunción denominada cintura hipertrigliceridémica $(\mathrm{CHT})^{10}$. Estudios indican que la utilización de CHT ayuda a identificar mejor a los pacientes con obesidad abdominal que pudieran estar desarrollando cierto grado de $\mathrm{RI}^{10}$.

Se conoce que los factores de riesgo cardiometabólico y las medidas antropométricas son influenciadas por diversas variables sociodemográficas, así como por la exposición a factores ambientales como la dieta. Considerando, además, que Venezuela atraviesa un proceso de transición nutricional, se planteó el presente trabajo que tuvo como objetivo evaluar el perfil metabólico de riesgo cardiovascular y RI en pacientes adultos según índice de masa corporal, circunferencia de cintura y cintura hipertrigliceridémica.

\section{Material y MÉTODO}

Estudio descriptivo, de corte transversal, que incluyó una muestra de 75 pacientes adultos, que consultaron un Ambulatorio Urbano de atención primaria de salud, Naguanagua, Estado Carabobo, Venezuela, en el período comprendido entre mayo y julio de 2008. Se solicitó consentimiento informado al paciente y contó con la aprobación del Comité de Ética del centro ${ }^{11}$. Se excluyó al adulto que presentase patología que pudiese alterar su peso o talla, con hipertensión arterial no controlada, diabetes mellitus, feocromocitoma $y$ terapia hipolipemiante.

Se midió peso, talla y CC, según protocolos internacionales recomendados ${ }^{12}$. En la determinación del peso y talla se utilizó una báscula previamente calibrada con estadímetro (Healthmeter) y la CC se midió con una cinta métrica no expansible, graduada en milímetros ( $\mathrm{mm})$. El IMC se estimó a partir de la relación peso (kg)/ talla $\left(\mathrm{m}^{2}\right)$, lo que permitió la distribución de los pacientes en tres grupos, normopeso (entre 18,5 y $24,9 \mathrm{~kg} / \mathrm{m}^{2}$ ), sobrepeso (entre 25 y $29,9 \mathrm{~kg}$ / $\mathrm{m}^{2}$ ) y obesos $\left(\geq 30 \mathrm{~kg} / \mathrm{m}^{2}\right)^{2}$. La CC y triglicéridos se clasificaron según los criterios de la $\operatorname{IDF}^{13}$, considerando CC elevada (mujeres $>80 \mathrm{~cm}$; hombres $>90 \mathrm{~cm}$ ) e hipertrigliceridemia $\geq 150$ $\mathrm{mg} / \mathrm{dL}$.

La presión arterial (PA) se midió utilizando el método auscultatorio, con un instrumento calibrado y adecuadamente validado. Los pacientes debieron estar sentados y quietos en una silla durante $5 \mathrm{~min}$, con los pies en el suelo, y el brazo a la altura del corazón ${ }^{14}$. 
Análisis de laboratorio. Se midió en muestra de ayunas glicemia y lípidos utilizando métodos colorimétricos enzimáticos (Wiener Lab) y para la insulina se utilizó el método ELISA (Crystal Chem Inc). La determinación de RI se realizó mediante el cálculo del índice HOMA (Homeostasis Model Assessment) ${ }^{15}$, que se calcula como insulina basal (mUI/ L) x glicemia basal $(\mathrm{mmol} / \mathrm{L}) / 22,5$. Se consideró como valor diagnóstico de RI un HOMA $>2,5^{16}$.

Estadística. Los datos se expresaron como media $(\bar{x}) \pm$ desviación estándar (DE). Se aplicó el test de Kolmogorov-Smirnov para conocer si las variables se distribuyeron en forma normal. Las comparaciones de variables continuas entre los pacientes normopeso, sobrepeso y obesidad se realizaron mediante análisis de la varianza (ANOVA) con la prueba post hoc de la máxima diferencia significativa de Tukey. Las comparaciones de los promedios de las variables según CC y CHT se realizaron a través de la t de Student para muestras independientes. Para establecer la contribución de múltiples variables independientes en la RI, se realizó un análisis multivariante mediante regresión lineal por selección de variables paso a paso (método stepwise). Se escogió un valor de p <0,05 como nivel de significación estadística.

\section{Resultados}

La edad promedio de los participantes fue de 46,1 $\pm 15,6$ años. De ellos, 63 (84\%) eran del género femenino (46,0 011,3 años) y 12 (16\%) del masculino (46,5 $\pm 10,2$ años). Ambos grupos fueron similares en cuanto a la distribución de la edad. El porcentaje de individuos con sobrepeso y obesidad fue de $37,3 \%$, respectivamente.

Los valores promedios de las variables estudiadas en el grupo total y según IMC se resumen en la Tabla 1. Al comparar entre categorías de IMC, se encontraron diferencias para las variables peso, IMC, CC, PA, lípidos, glicemia, insulina y HOMA a medida que se incrementó el IMC.

De los pacientes estudiados, $80 \%$ presentó CC elevada y 50,6\% CHT. En los individuos con CC

Tabla 1. Valores representativos (media \pm desviación estándar) de las variables estudiadas, en el grupo total y según índice de masa corporal

\begin{tabular}{|lccccc|}
\hline & $\begin{array}{c}\text { Grupo total } \\
\mathbf{n = 7 5}\end{array}$ & $\begin{array}{c}\text { Normopeso } \\
\mathbf{n = 1 9}\end{array}$ & $\begin{array}{c}\text { Sobrepeso } \\
\mathbf{n = 2 8}\end{array}$ & $\begin{array}{c}\text { IMC } \\
\text { Obesidad } \\
\mathbf{n = 2 8}\end{array}$ & $\mathbf{p}$ \\
\hline Peso (kg) & $72,26 \pm 15,8$ & $56,39 \pm 5,52^{\mathrm{a}}$ & $68,45 \pm 7,30^{\mathrm{b}}$ & $86,84 \pm 14,04^{\mathrm{c}}$ & 0,000 \\
Talla (mt) & $15,8 \pm 0,07$ & $1,58 \pm 0,07$ & $1,59 \pm 0,08$ & $1,57 \pm 0,07$ & 0,726 \\
IMC & $29,19 \pm 6,18$ & $22,58 \pm 1,31^{\mathrm{a}}$ & $27,10 \pm 1,38^{\mathrm{b}}$ & $35,76 \pm 4,64^{\mathrm{c}}$ & 0,000 \\
CC (cm) & $94,35 \pm 14,6$ & $79,42 \pm 4,89^{\mathrm{a}}$ & $92,11 \pm 7,63^{\mathrm{b}}$ & $106,71 \pm 13,78^{\mathrm{c}}$ & 0,000 \\
PAS (mmHg) & $120,07 \pm 18,20$ & $114,47 \pm 17,71^{\mathrm{a}, \mathrm{b}}$ & $116,07 \pm 14,99^{\mathrm{b}}$ & $127,86 \pm 19,31^{\mathrm{c}}$ & 0,014 \\
PAD (mmHg) & $76,80 \pm 11,53$ & $73,68 \pm 13,42^{\mathrm{a}, \mathrm{b}}$ & $74,64 \pm 9,22^{\mathrm{b}}$ & $81,07 \pm 11,33^{\mathrm{c}}$ & 0,043 \\
Col Total (mg/dL) & $211,08 \pm 35,84$ & $197,21 \pm 32,67^{\mathrm{a}, \mathrm{b}}$ & $201,21 \pm 38,72^{\mathrm{b}}$ & $230,46 \pm 25,86^{\mathrm{c}}$ & 0,002 \\
HDLc (mg/dL) & $39,45 \pm 4,44$ & $41,16 \pm 4,71^{\mathrm{a}, \mathrm{b}}$ & $40,43 \pm 3,11^{\mathrm{b}}$ & $37,32 \pm 4,67^{\mathrm{c}}$ & 0,004 \\
LDLc (mg/dL) & $140,52 \pm 34,76$ & $131,05 \pm 33,46^{\mathrm{a}, \mathrm{b}}$ & $131,11 \pm 39,18^{\mathrm{b}}$ & $156,36 \pm 34,80^{\mathrm{c}}$ & 0,018 \\
TGL (mg/dL) & $164,09 \pm 56,95$ & $130,26 \pm 23,27^{\mathrm{a}, \mathrm{b}}$ & $153,79 \pm 46,01^{\mathrm{b}}$ & $197,36 \pm 65,93^{\mathrm{c}}$ & 0,000 \\
Glicemia (mg/dL) & $88,96 \pm 9,84$ & $88,05 \pm 9,17^{\mathrm{a}, \mathrm{b}}$ & $84,71 \pm 6,92^{\mathrm{b}}$ & $93,82 \pm 10,86^{\mathrm{c}}$ & 0,002 \\
Insulina (mUI/L) & $12,82 \pm 3,71$ & $9,28 \pm 1,23^{\mathrm{a}}$ & $12,29 \pm 2,57^{\mathrm{b}}$ & $15,76 \pm 3,47^{\mathrm{c}}$ & 0,000 \\
RI (HOMA) & $2,86 \pm 1,03$ & $2,01 \pm 0,33^{\mathrm{a}}$ & $2,59 \pm 0,65^{\mathrm{b}}$ & $3,69 \pm 1,04^{\mathrm{c}}$ & 0,000 \\
\hline
\end{tabular}

CC: Circunferencia de cintura; PAS: Presión arterial sistólica; PAD: Presión arterial diastólica; Col total: Colesterol total; HDLc: Colesterol unido a la lipoproteína de alta densidad; LDLc: Colesterol unido a la lipoproteína de baja densidad; TGL: Triglicéridos; HOMA: Homeostasis Model Assessment.

Letras diferentes ( $\mathrm{a}, \mathrm{b}$ y c) indican promedios que difieren significativamente entre categorías de IMC. 
elevada se evidenció mayor peso, IMC, PAS, triglicéridos, insulina y HOMA con respecto a aquellos con CC normal. Los pacientes identificados con CHT presentaron adicionalmente un aumento significativo de PAD, glicemia, colesterol total y disminución de la HDLc en relación con el grupo sin CHT (Tabla 2); resultados idénticos se obtuvieron cuando se compararon los individuos con CHT vs individuos con sólo CC elevada.

Según el análisis multivariante de regresión lineal se observó un efecto positivo del IMC, la CHT y la PAD sobre la RI ( $p<0,001)$ (Tabla 3$)$.

\section{DisCusión}

Los resultados del presente estudio revelan una importante frecuencia de exceso de peso y de obesidad abdominal, indicando que el sobrepeso constituye un factor de riesgo de alta prevalencia en el municipio investigado, situación que puede ser atribuida al hecho de que países como Venezuela, están adoptando hábitos sedentarios y dietas con alto contenido en grasas saturadas y carbohidratos $^{17}$. La prevalencia observada concuerda con la encontrada en Paraguay $(70 \%)^{17}$,

Tabla 2. Valores representativos (media \pm desviación estándar) de las variables estudiadas, según circunferencia de cintura (CC) y cintura hipertrigliceridémica (CHT)

\begin{tabular}{|c|c|c|c|c|c|c|}
\hline & $\begin{array}{c}\text { CC } \\
\text { normal } \\
n=15\end{array}$ & $\begin{array}{c}\text { CC } \\
\text { elevada* } \\
\mathbf{n}=\mathbf{6 0}\end{array}$ & $\mathbf{p}$ & $\begin{array}{c}\text { CHT } \\
\text { ausente } \\
\mathbf{n}=37\end{array}$ & $\begin{array}{c}\text { CHT } \\
\text { presente }^{\dagger} \\
\mathbf{n}=38\end{array}$ & $\mathbf{p}$ \\
\hline Peso (kg) & $56,29 \pm 5,55$ & $76,25 \pm 14,94$ & 0,000 & $63,44 \pm 10,14$ & $80,86 \pm 15,66$ & 0,000 \\
\hline Talla (m) & $1,57 \pm 0,05$ & $1,58 \pm 0,08$ & 0,494 & $1,59 \pm 0,07$ & $1,56 \pm 0,07$ & 0,142 \\
\hline $\operatorname{IMC}\left(\mathrm{Kg} / \mathrm{m}^{2}\right)$ & $22,85 \pm 1,86$ & $30,77 \pm 5,86$ & 0,000 & $24,93 \pm 3,08$ & $33,34 \pm 5,60$ & 0,000 \\
\hline $\mathrm{CC}(\mathrm{cm})$ & $77,13 \pm 2,92$ & $98,65 \pm 13,09$ & 0,000 & $86,16 \pm 9,88$ & $102,32 \pm 14,12$ & 0,000 \\
\hline PAS (mmHg) & $110,67 \pm 12,23$ & $122,42 \pm 18,77$ & 0,024 & $113,92 \pm 15,86$ & $126,05 \pm 18,53$ & 0,003 \\
\hline PAD (mmHg) & $72,00 \pm 8,62$ & $78,00 \pm 11,90$ & 0,071 & $72,70 \pm 11,46$ & $80,79 \pm 10,24$ & 0,002 \\
\hline Col Total $(\mathrm{mg} / \mathrm{dL})$ & $201,67 \pm 33,01$ & $213,43 \pm 36,39$ & 0,258 & $201,24 \pm 33,11$ & $220,66 \pm 36,21$ & 0,018 \\
\hline HDLc (mg/dL) & $40,73 \pm 4,45$ & $39,13 \pm 4,41$ & 0,214 & $40,70 \pm 3,88$ & $38,24 \pm 4,65$ & 0,015 \\
\hline LDLc (mg/dL) & $136,00 \pm 34,12$ & $141,65 \pm 35,11$ & 0,577 & $134,38 \pm 33,40$ & $146,50 \pm 35,45$ & 0,132 \\
\hline TGL (mg/dL) & $131,53 \pm 23,00$ & $172,23 \pm 60,04$ & 0,000 & $137,62 \pm 31,25$ & $189,87 \pm 64,44$ & 0,000 \\
\hline Glicemia $(\mathrm{mg} / \mathrm{dL})$ & $85,73 \pm 8,36$ & $89,77 \pm 10,08$ & 0,157 & $84,30 \pm 8,32$ & $93,50 \pm 9,14$ & 0,000 \\
\hline Insulina (mUI/L) & $9,23 \pm 1,36$ & $13,72 \pm 3,57$ & 0,000 & $9,93 \pm 1,67$ & $15,63 \pm 2,89$ & 0,000 \\
\hline HOMA & $1,95 \pm 0,30$ & $3,08 \pm 1,02$ & 0,000 & $2,06 \pm 0,39$ & $3,63 \pm 0,85$ & 0,000 \\
\hline
\end{tabular}

"CC elevada en hombres: CC $>90 \mathrm{~cm}$; en mujeres CC $>80 \mathrm{~cm}$.

${ }^{\dagger}$ CHT en hombres: CC $>90 \mathrm{~cm}$ e hipertrigliceridemia $\geq 150 \mathrm{mg} / \mathrm{dL}$; en mujeres CC $>80 \mathrm{~cm}$ e hipertrigliceridemia $\geq 150 \mathrm{mg} / \mathrm{dL}$.

Tabla 3. Predicción de la resistencia a la insulina determinado por HOMA (H omeostasis M odel Assesment), mediante regresión lineal*

\begin{tabular}{|lcrc|}
\hline Variables predictivas & $\boldsymbol{B}$ & $\mathbf{t}$ & $\mathbf{p}$ \\
\hline Constante & $-1,655$ & $-3,475$ & 0,001 \\
IMC $\left(\mathrm{kg} / \mathrm{m}^{2}\right)$ & 0,427 & 4,951 & 0,000 \\
CHT $(\mathrm{cm}-\mathrm{mg} / \mathrm{dL})$ & 0,415 & 4,740 & 0,000 \\
PAD $(\mathrm{mm} \mathrm{Hg})$ & 0,171 & 2,537 & 0,013 \\
\hline
\end{tabular}

Resumen del modelo: $\mathrm{R}=0,850 ; \mathrm{F}=61,558 ; \mathrm{p}<0,001$.

*Variables independientes: edad, género, IMC, CC, CHT, PAS, PAD, colesterol total, HDLc, LDLc y triglicéridos. 
seguida de Estados Unidos de Norteamérica y Chile con $64 \%$ y $61,3 \%$, respectivamente ${ }^{18}$.

En esta investigación, a medida que se incrementó el IMC, existió tendencia al aumento de los niveles de colesterol total, LDLc y triglicéridos, con una reducción de la HDLc, encontrándose diferencias significativas cuando se comparó el grupo obeso versus el normopeso o el grupo obeso versus el sobrepeso. Esta dislipidemia se acompañó de elevación significativa de la insulina y del índice HOMA, presentando mayor grado de RI los pacientes obesos, lo cual coincide con lo reportado por otros autores ${ }^{19}$. En el grupo con sobrepeso, sólo la insulina y el HOMA se incrementaron significativamente con respecto al grupo normopeso. Lo anterior denota la importancia de la RI como hallazgo común al acúmulo progresivo de grasa corporal. En concordancia con los resultados obtenidos, se ha propuesto que la disminución de la sensibilidad a la insulina asociada a la obesidad obedece a que la funcionalidad del tejido adiposo es afectada por su hipertrofia y sobre todo por su acúmulo en el abdomen, convirtiéndose en un tejido inflamado, infiltrado anormalmente por macrófagos, que hipersecreta leptina, citoquinas y resistina; estos agentes humorales se han relacionado con RI debido a que pueden alterar las vías de señalización de la hormona ${ }^{20}$. Adicionalmente, disminuye su secreción de adiponectina, una adipoquina a la cual se han atribuido efectos insulino-sensibilizadores. El tejido adiposo hipertrófico también libera una elevada cantidad de ácidos grasos libres, los cuales contribuyen al establecimiento de la RI con una hiperinsulinemia compensatoria $^{20,21}$.

Diversos estudios demuestran una asociación significativa entre obesidad intraabdominal, el riesgo de enfermedad cardiovascular y diabetes ${ }^{22}$. Al distribuir los pacientes de acuerdo a su CC, se evidenciaron valores superiores de peso, IMC, PAS, triglicéridos, insulina y HOMA en los individuos con CC elevada. Si bien es cierto que no se alcanzaron diferencias estadísticamente significativas para PAD y otros indicadores del perfil lipídico, la tendencia de dichas variables fue similar a la observada en los individuos obesos según IMC. Farin et $\mathrm{al}^{23}$ evidenciaron en adultos sanos que a mayor adiposidad mayor es la RI, glicemia y trigliceridemia, independientemente del indicador de adiposidad empleado, sea IMC O CC; sin embargo, estos autores sólo observaron incremento del colesterol total y LDLc, así como disminución de HDLc cuando se definió obesidad por IMC. Aunque en el presente trabajo el número de individuos por grupo pudo impedir probar diferencias y los resultados coinciden con los reportados por Farin et $\mathrm{al}^{23}$, es importante destacar que el IMC promedio en el grupo con CC elevada fue menor que el de los individuos obesos según IMC. A este respecto, la gordura per se, sin separarla del grado de obesidad (sobrepeso contra obesidad) o distribución (central vs periférica) exhibe un efecto dosis-respuesta sobre los lípidos sanguíneos ${ }^{24}$.

La dislipidemia observada en los individuos obesos según IMC o con obesidad abdominal según CC corresponde a la descrita por la literatu$\mathrm{ra}^{25}$. El perfil lipídico de la obesidad se caracteriza por hipertrigliceridemia y valores bajos de HDLc $^{25}$. En relación al colesterol total y LDLc, por lo general, se incrementan ligeramente o se mantienen dentro de los límites normales, lo cual concuerda con lo encontrado en este trabajo. Los mecanismos metabólicos que explican los cambios observados se asocian a RI y han sido ampliamente revisados ${ }^{25,26}$.

La PA promedio se elevó con el grado de obesidad y el incremento de la CC. La obesidad está estrechamente relacionada con valores de PA por encima de lo óptimo, siendo mayores cuando la obesidad es abdominal. El aumento de la PA podría deberse a efectos directos sobre la hemodinamia o a incremento de la resistencia vascular periférica debido a disfunción endotelial, RI, activación del sistema nervioso simpático, adipoquinas o apnea del sueño ${ }^{27}$. La glicemia, por su parte, siguió la misma tendencia que la PA, aunque no se observaron valores promedios diagnósticos de intolerancia a la glucosa o diabetes. Se ha probado que la función de la célula $\beta$ pancreática se reduce antes de que se demuestre hiperglicemia, ocurriendo una transición entre una tolerancia normal a la glucosa a una anormal, hasta llegar a diabetes ${ }^{28}$. Si bien la diabetes es más frecuente entre los individuos obesos, sólo una pequeña porción de ellos la desarrolla. Uno de los mecanismos planteados que explicaría la pérdida de la función de la célula $ß$ sería la RI, la cual incrementaría su función secretora, resultando una célula "exhausta" a largo plazo ${ }^{28}$. 
Se evidenció una alta proporción de pacientes con CHT $(50,6 \%)$, esta prevalencia es notablemente mayor a la hallada entre hombres canadienses ${ }^{29}$ y mujeres norteamericanas ${ }^{30}$, las diferencias podrían deberse a las características de las poblaciones estudiadas y al tamaño de la muestra. Por otra parte, en comparación con los individuos sin CHT y también con aquellos que sólo presentaron CC elevada, los pacientes con CHT presentaron mayor peso, IMC, CC, PAS, PAD, colesterol total, triglicéridos, glicemia, insulina y RI así como cifras más bajas de HDLc. Estos resultados son semejantes a los encontrados en adultos norteamericanos $^{31}$ e iraníes ${ }^{32}$ donde se evidenció un perfil metabólico más adverso entre los individuos con CHT, apoyando la propuesta que la CHT es un fenotipo útil para identificar pacientes con exceso de tejido adiposo visceral, con acumulación ectópica de grasa en músculo, epicardio e hígado ${ }^{10}$ y a riesgo de desarrollar síndrome metabólico 33,34 . A la par, diversos estudios han demostrado el valor predictivo de la CHT para enfermedad cardiovascular en individuos con y sin alteración del metabolismo de la glucosa 35,36 .

Kahn y Valdez ${ }^{31}$ compararon los cambios metabólicos que se detectan en individuos con CHT alterada vs individuos con IMC $\geq 30 \mathrm{~kg} / \mathrm{m}^{2}$, observando mayor dislipidemia y glicemia entre los pacientes con CHT pero mayor insulinemia y PAS en los pacientes con sólo IMC elevado. En la presente investigación se encontró que los valores medios de CC, PA y lípidos en los pacientes obesos fueron más desfavorables, sin embargo, la glicemia, insulinemia y HOMA promedios fueron similares según IMC y CHT. Es preciso destacar que los individuos con CHT mostraron un IMC y CC promedio menores que los pacientes obesos según IMC, por lo que esta diferencia podría explicar parcialmente las cifras más adversas de lípidos y PA en los obesos. No obstante, también pudiera inferirse que la CHT podría ser un indicador igualmente sensible para detectar hiperinsulinemia y RI como el IMC, ya que el grupo de pacientes con CHT aun con valores medios de IMC menores presentaron cifras comparables de tales variables.

En este trabajo se demostró un efecto positivo del IMC, la CHT y la PAD sobre la RI. La influencia del IMC sobre la RI ha hecho pensar a muchos investigadores que el factor ambiental cumple un rol fundamental, donde la predisposición genética, hábitos alimentarios inadecuados, la disminución de la actividad física y los efectos de la urbanización, especialmente en los países en desarrollo, juegan un papel preponderante ${ }^{37}$. Ascaso et al ${ }^{38}$ reportaron una correlación significativa de la RI con el perímetro de cintura y los triglicéridos plasmáticos. Santi et $\mathrm{al}^{3}$ hallaron resultados similares. Por otra parte, varios mecanismos relacionan la RI con la hipertensión, estableciéndose que la insulina estimula la producción de endotelina-1 y la acción de ésta en la pared vascular; así mismo promueve la reabsorción renal de sodio, relacionándose la RI con hipertensión arterial sal sensible ${ }^{39}$.

Por último, es preciso señalar que el presente estudio tiene varias limitaciones. El diseño transversal del mismo impide inferir relaciones causales. Por el tamaño y predominio del género femenino en la muestra así como por el modo en que se enrolaron los individuos estudiados no fue posible distribuir los datos según género o edad, ni es posible realizar extrapolaciones poblacionales.

En conclusión, los pacientes con CHT demostraron un perfil cardiometabólico más desfavorable que el encontrado con CC elevada, aunque no más que el evidenciado de acuerdo al IMC. La CHT podría incluirse en la práctica clínica como una herramienta para identificar individuos asintomáticos de alto riesgo metabólico, más allá de la utilización del IMC y de la CC como únicas variables. Se requieren estudios prospectivos que corroboren los resultados obtenidos.

\section{Agradecimientos}

A todos los pacientes que participaron en el estudio. A docentes y estudiantes de la Escuela de Bioanálisis de la Universidad de Carabobo, que formaron parte del Proyecto de Servicio Comunitario "Integración Comunidad-Universidad para el análisis de la problemática de la Hipertensión Arterial en adultos de comunidades de Naguanagua, Estado Carabobo, como base para diseñar proyectos sociales". Año 2008.

Al personal de Salud del Ambulatorio "Nuestra Señora de la Luz". Facultad de Ciencias de la Salud, Universidad de Carabobo. 


\section{Referencias}

1. SAltiel AR, KaHm CR. Insulin signalling and the regulation of glucose and lipid metabolism. Nature 2001; 414: 799-806.

2. World Health Organization. Physical status: the use and interpretation of anthropometry. Report of a WHO Expert Committee. WHO Technical Report Series 854. Geneva: World Health Organization; 1995.

3. Santi MJ, Carrozas A, Barba A, Astola A, Jiménez A, Mangas A. Circunferencia de la cintura como predictor de resistencia insulínica en varones jóvenes. Med Clin 2005; 125: 46-50.

4. Molist N, Jimeno J, Franch J. Correlación entre las diferentes medidas de obesidad y el grado de resistencia a la insulina. Aten Primaria 2006; 37: 30-6.

5. Després JP, Poirier P, Bergeron J, Tremblay A, Lemieux I, Alméras N. From individual risk factors and the metabolic syndrome to global cardiometabolic. Eur Heart J 2008; 10: B24-B33.

6. Barter P, McPherson YR, Song K, Kesaniemi YA, Mahley R, Waeber G ET AL. Serum Insulin and Inflammatory Markers in Overweight Individuals with and without Dyslipidemia. J Clin Endocrinol Metab 2007; 92: 2041-5.

7. Vague J. La différenciation sexuelle, facteur déterminant des formes de l'obésité. Presse Médicale 1947; 30: 339-40.

8. Després JP. Health consequences of visceral obesity. Annals of Medicine 2001; 33: 534-41.

9. Lamarche B, Tchernof A, Mauriege P, Cantin B, Dagenais G, Lupien P ET AL. Fasting insulin and apolipoprotein B levels and low-density lipoprotein particle size as risk factors for ischemic heart disease. JAMA 1998; 279: 1955-61.

10. Lemieux I, Pascot A, Coulllard C, Lamarche B, Tchernof A, Almeras $\mathrm{N}$ et al. Hypertriglyceridemic waist: A Marker of the Atherogenic Metabolic Triad (Hyperinsulinemia; Hyperapolipoprotein B; Small, Dense LDL) in Men? Circulation 2000; 102: 179-84.

11. De Roy PG. Helsinki and the Declaration of Helsinki. World Med J 2004; 50(1): 9-11).

12. Lohman TG, Roche AF, Martorell R. Anthropometric standardization reference manual. Champaign, IL: Human Kinetics Books; 1988.

13. International Diabetes Federation (IDF). Worldwide definition of Metabolic Syndrome. Rev Panam Salud Pública 2005; 18: 665-9.

14. Joint National Committee on Prevention, Detection, Evaluation, and Treatment of High Blood Pressure. The Seventh Report of the Joint National Committee on Prevention, Detection, Evaluation, and Treatment of High Blood Pressure. JAMA 2003; 289: 2560-72.

15. Matthews D, Hosker J, Rudenski A, Naylor B, Teacher D, Turner R. Homeostasis model assessment insulin resistance and cell function from fasting plasma glucose and insulin concentration in man. Diabetologia 1985; 28: 412-9.

16. Sociedad Venezolana de Endocrinología y Metabolismo. Consenso Nacional de Diabetes Mellitus Tipo 2, Venezuela. Caracas: Editorial Traducciencia; 2003.

17. Barría RM, Amigo H. Transición Nutricional: Una revisión del perfil Latinoamericano. ALAN 2006; 56: 03-11.

18. Vıo F. Prevención de la obesidad en Chile. Rev Chil Nutr 2005; 32: 80-7.

19. Mclaughlin T, Allison G, Abbasi F, Lamendola C, Reaven G. Prevalence of insulin resistance and associated cardiovascular disease risk factors among normal weight, overweight, and obese individuals. Metabolism 2004; 53: 495-9.

20. Bastard JP, Maachi M, Lagathu C, Kim MJ, Caron M, Vidal H, Capeau J, Feve B. Recent advances in the relationship between obesity, inflammation, and insulin resistance. Eur Cytokine Netw 2006; 17: 4-12.

21. Vázquez-Vela ME, Torres N, Tovar AR. White adipose tissue as endocrine organ and its role in obesity. Arch Med Res 2008; 39: 715-28.

22. Klein S, Aluison DB, Heymsfield SB, Kelley DE, Leibel RL, Nonas C, Kahn R; Association for Weight Management and Obesity Prevention; NAASO, The Obesity Society; American Society for Nutrition; American Diabetes Association. Waist circumference and cardiometabolic risk: a consensus statement from Shaping America's Health: Association for Weight Management and Obesity Prevention; NAASO, The Obesity Society; the American Society for Nutrition; and the American Diabetes Association. Am J Clin Nutr 2007; 85: 1197-202.

23. Farin HM, Abbasi F, Reaven GM. Comparison of body mass index versus waist circumference with the metabolic changes that increase the risk of cardiovascular disease in insulin-resistant individuals. Am J Cardiol 2006; 98: 1053-6.

24. Troya-Barriga P. Dislipidemia y Obesidad. Gac Méd Méx 2004; 140 Suplement 2: S49- S58.

25. Franssen R, Monajemi H, Stroes es, Kastelein JJ. Obesity and dyslipidemia. Endocrinol Metab Clin North Am 2008; 37: 6236-6233.

26. Mooradian AD, HaAs MJ, Wehmeier KR, Wong NC. Obesity-related changes in high-density lipoprotein metabolism. Obesity (Silver Spring) 2008; 16: $1152-$ 60.

27. Poirier P, Giles TD, Bray GA, Hong Y, Stern JS, PiSunYER FX, EcKel RH; American Heart Association; Obesity Committee of the Council on Nutrition, Physical Activity, and Metabolism. Obesity and cardiovascular disease: pathophysiology, evaluation, and effect of weight loss: an update of the 1997 American Heart Association Scientific Statement on Obesity and Heart Disease from the Obesity Com- 
mittee of the Council on Nutrition, Physical Activity, and Metabolism. Circulation 2006; 113: 898-918.

28. KaHn SE. Clinical review 135: The importance of beta-cell failure in the development and progression of type 2 diabetes. J Clin Endocrinol Metab 2001; 86: 4047-58.

29. Lemieux I, Alméras N, Mauriège P, Blanchet C, Dewailly E, Bergeron J, Després JP. Prevalence of 'hypertriglyceridemic waist' in men who participated in the Quebec Health Survey: association with atherogenic and diabetogenic metabolic risk factors. Can J Cardiol 2002; 18: 725-32.

30. Lamonte Mj, Ainsworth Be, Dubose KD, Grandjean PW, Davis PG, Yanowitz FG, Durstine JL. The hypertriglyceridemic waist phenotype among women. Atherosclerosis 2003; 171: 123-30.

31. Kahn HS, Valdez R. Metabolic risks identified by the combination of enlarged waist and elevated triacylglycerol concentration. Am J Clin Nutr 2003; 78: 92834.

32. Solati M, Ghanbarian A, Rahmani M, Sarbazi N, Allahverdian S, Azizi F. Cardiovascular risk factors in males with hypertriglycemic waist (Tehran Lipid and Glucose Study). Int J Obes Relat Metab Disord 2004; 28: 706-9.

33. Gazi IF, Filippatos TD, Tsiminodimos V, Saougos VG, Liberopoulos EN, Mikhailidis DP ET al. The hypertriglyceridemic waist phenotype is a predictor of elevated levels of small, dense LDL cholesterol. Lipids 2006; 41: 647-54.

34. Després JP, Cartier A, Côtém, Arsenault BJ. The concept of cardiometabolic risk: Bridging the fields of diabetology and cardiology. Annals of Medicine 2008; 1-10.

35. Czernichow S, Bruckert E, Bertrais S, Galan P, Hercberg S, Oppert JM. Hypertriglyceridemic waist and 7.5-year prospective risk of cardiovascular disease in asymptomatic middle-aged men. Int J Obes (Lond) 2007; 31: 791-6.

36. St-Pierre J, Lemieux I, Perron P, Brisson D, Santure M, VOHL MC ET AL. Relation of the hypertriglyceridemic waist' phenotype to earlier manifestations of coronary artery disease in patients with glucose intolerance and type 2 diabetes mellitus. Am J Cardiol 2007; 99: 369-73.

37. Maes HH, Neale MC, Eaves LJ. Genetic and environmental factors in relative body weight and human adiposity. Behav Genet 1997; 27: 325-51.

38. Ascaso JF, Romero P, Real JT, Lorente Ri, Martínez J, CARMENA R. Abdominal adiposity (waist circumference) and its relation to insulin resistance and the metabolic syndrome in a South European Population. Eur J Intern Med 2003; 14: 101-6.

39. Yanai H, Tomono Y, Ito K, Furutani Y, Yoshida $H$, TADA N. Review: The underlying mechanisms for development of hypertension in the metabolic syndrome. Nutrition Journal 2008; 7: 10. 